\title{
Building Feature Extraction via a Deterministic Approach: Application to Real High Resolution SAR Images
}

\author{
Giorgio Franceschetti, Raffaella Guida, \\ Antonio Iodice, Daniele Riccio, Giuseppe Ruello \\ DIET \\ Università Federico II \\ Napoli, Italy \\ \{gfrance, rafguida, iodice, daniele.riccio, ruello\}@unina.it
}

\author{
Uwe Stilla \\ Photogrammetry \& Remote Sensing \\ Technische Universität \\ München, Germany \\ stilla@tum.de
}

\begin{abstract}
Interpretation of high resolution SAR (Synthetic Aperture Radar) images is still a hard task, especially when manmade objects crowd the scene under detection. This paper contributes to the analysis of this kind of data by adopting an approach, based on a scattering model, for the retrieval of buildings height from real SAR images and presenting first numerical results.
\end{abstract}

Keywords: Synthetic Aperture Radar (SAR), high resolution, urban areas.

\section{INTRODUCTION}

The analysis and interpretation of real SAR (Synthetic Aperture Radar) images of urban areas are still an open issue. The presence of distortion effects as well as multiple scattering due to dihedral and trihedral geometries, typical of built-up areas, makes the understanding of relevant SAR images hard.

Despite of this, improvements in SAR technology will soon provide clearer images, even on urban centers, thanks to the high spatial resolution (till 1 meter) distinctive of new forthcoming sensors, like the German TerraSAR-X [1]. Consequently, also our capability of retrieving detailed information, in a quantitative sense, about objects in the urban scene is considerably improving.

Recently, some new approaches for feature extraction from high resolution SAR images of urban areas have been presented [2],[3].

On one side, the strength of these approaches has been demonstrated to be the fact that they are model-based, meaning that a geometrical model of the urban scene and a sound scattering model for the estimation of signal return towards the radar have been previously introduced [4] and then adopted for the feature extraction [2]. This way of representing the reality is really powerful as only model-based approaches can lead to quantitatively analyze the SAR image and, consequently, to quantitatively retrieve relevant information. In fact, by means of such approaches, we get a system of equations linking, through the models adopted, the geometric and electromagnetic scene parameters and the radar ones to the parameters measurable on the SAR image (radar cross section, the extension of layover and shadow areas, etc..). These relationships, even if not always linear or simply invertible, lead to a quantitative extraction of the desired unknowns.

But, on the other side, this ability has to reckon with a high a-priori knowledge of the radar and scene parameters needed for the application of these methods. In [3], for example, the application of the so called deterministic approach proposed in [2] for retrieving the building height from radiometric parameters has been shown to strongly depend upon a previous knowledge, among the others, of soil roughness parameters and the radar flight trajectory.

In order to foresee the efficiency of an application of the above retrieval approach on real SAR images, in [2],[3] the method has been first tested on simulated SAR images relevant to canonical urban scenes, thus highlighting the guiding principles for an experiment on real SAR images.

Those guidelines are followed in this paper in which the deterministic approach for feature extraction from high resolution SAR images of urban areas is, for the first time in literature, applied and tested on real SAR images. In particular, in Section II the rationale applied for the building height retrieval is explained. In Section III, the visit of the site and the collected information are described to support the application of the extraction algorithm detailed in Section IV. Results and limits are finally discussed.

\section{RATIONALE}

The feature extraction that the authors carry on concerns the retrieval of a building height $h$ from its double reflection contribution to the radar cross section $\sigma^{o}$ according to the inverted relationship presented in [3]:

$$
h=\left|\sigma^{\circ}\right| \frac{8 \pi^{2} \cos ^{2} \vartheta \cdot \sigma^{2}\left(2 / L^{2}\right) \cdot \exp \left[\frac{\tan ^{2} \vartheta \sin ^{2} \varphi}{2 \sigma^{2}\left(2 / L^{2}\right)}\right]}{\left|S_{p q}\right|^{2} l \tan \vartheta \cos \varphi\left(1+\tan ^{2} \vartheta \sin ^{2} \varphi\right)}
$$


where $l$ is the length of the building, $\sigma$ and $L$ are, respectively, standard deviation and correlation length of the stochastic process describing the roughness of the soil on which the building is placed, $S_{p q}$ is the generic element of the scattering matrix, with $p$ and $q$ each standing for $h$ or $v$ (horizontal or vertical polarization), $\theta$ is the radar look angle and $\varphi$ is the building wall orientation.

Equation (1) can be considered to well represent the scattering relevant to the double reflection if the Geometric Optics (GO) approximation of Kirkhhoff is valid for both bounces. This is verified when the soil surfaces involved are rough compared to the working wavelength.

In this paper, (1) is applied on a real SAR image acquired on 20 April 2004 by an airborne SAR sensor in the X-band during a flight campaign concerning the area of Oberpfaffenhofen.

We work on the image in different steps: we first process the image with a byte scale operation for a correct visualization of the double reflection lines. After localizing the contributions relevant to the selected buildings (those ones satisfying the requirements of the approach) we move to the original SAR image to exploit all the radiometric resolution and from this we extract the mean grey levels of double reflections.

At this point, since the considered SAR image is not radiometrically corrected, we have to perform a calibration step. For this operation we generally need to know two buildings heights to make the correct correspondence between all the heights in the image and their relevant contributions to the radar cross section in terms of grey levels. In this particular case we show that the a-priori knowledge of the geometry of only one building could be sufficient for the calibration. For this building the complete geometry will be retrieved mainly adopting the brightness of its double reflection contributions (more than one for its particular shape) and their positions in the SAR image, as explained in Section IV.

Some comments are in order to explain the main difficulties met in the retrieval procedure and some simplified approximations assumed in Section IV.

Working at the SAR image, the authors appreciated the presence of many bright lines. For a correct application of their feature extraction they had to distinguish the lines relevant to double bounces from those coming from other effects. Geometrical considerations, based on the expected localization of such contributions, allowed to carry on this distinction.

The approximation of GO for both bounces can be adopted when the soil roughness satisfies $2 \pi \sigma / \lambda » 1$, where $\lambda$ is the working wavelength, and the building wall is smooth.

As Fig.1 shows, the ground in front of the buildings is mainly constituted by grass and asphalt. Being the working frequency $f=9.6 \mathrm{GHz}$, the roughness hypothesis is verified for $\sigma \gg 5 \mathrm{~mm}$. This is widely fulfilled by the grass but not by the asphalt whose imperfections are in the order of some millimetres.

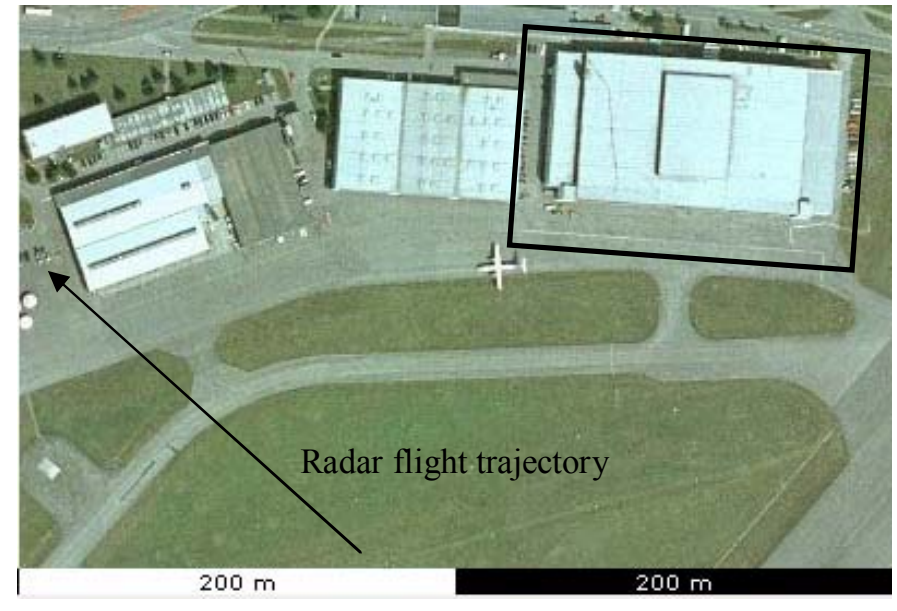

Figure 1. Airborne optical image of the airport area of DLR in Oberpfaffenhofen. (GeoContent $\mathrm{GmbH}$ )

In this particular case, the adoption of Physics Optics (PO) in the Kirkhhoff approach would have not improved the scattering model as now both the roughness of grass and asphalt would not satisfy the hypothesis of PO. At the moment the authors are working on this aspect to evaluate the consequences of adopting a scattering model whose hypothesis are not completely fulfilled.

Application of (1) asks for the knowledge of many parameters describing the scene. These have been mainly got by visiting the site described in the next section.

\section{DESCRIPTION OF THE SITE}

The site to be studied, see Fig.1, has been individuated in the airport area of the German Aerospace Center (DLR), located in a suburban area of the little village of Oberpfaffenhofen, near Munich, where buildings quite well approximated by the geometric model adopted by the deterministic approach, [4], have been found. Actually, the buildings have tilted roofs while the inversion procedure models them as parallelepipeds, but their slope is so low that, for the extraction carried on in the following, the approximation of flat roof can be accepted and a mean value for the heights involved has been reasonably adopted.

Also the scattering model in [4] seems to fit what happens in the real scene, being the buildings chosen for the test quite isolated in an electromagnetic sense. In fact, if we consider the radar flight trajectory in the SAR image, added to the optical image in Fig. 1 for simplicity, the main facades of the selected structures, have free areas in front of them. In this way the signal coming from the sensor is not caught by complex geometries but it goes back to the radar with some information that is quite well represented by the equations introduced in [4] and here inverted for the extraction at issue. But we are interested also in the short sides of the buildings and for these the hypothesis of open space in front could not be satisfied. For the bigger building on the top right side in the black frame, considered for our retrieval, this is verified also for the short side. We measured in fact the distance between this side and 
the neighbouring building verifying that it is wider than the extension of the ground area involved by the double bounces (depending on the heights of the buildings and the radar look angle). This means that, according to the scattering model, the double reflection from this side is related to the all building height and not to a part.

The site has been visited and studied in order to collect as much information as possible about the buildings in the scenes (the geometric dimensions, the materials of the front walls, the materials of soil on which the buildings are placed and its roughness) in order to get the a-priori knowledge required by the deterministic method. Despite of this, some information is still missing but here the authors go around the problem exploiting the results of sensibility analysis presented in [3]. In particular, we chose a building in the scene for which the sensitiveness to unknown parameters is low according to the analysis in [3].

Let us concentrate on this one. The geometry of this building looks like that of three boxes, one above the other. The length and width of each part has been retrieved by the optical image in Fig. 1 and are reported in meters in the enlarged view in Fig.2.

The heights of the different parts have been also deduced by optical images taken on the site and are resumed in Table 1.

The presence of three different heights along the short side of the building suggested us to use two of them (relevant to the central and upper part) for radiometrically calibrate the SAR image and the third one (relevant to the lower part) for the extraction. We underline that in the feature extraction from the SAR image, we compare the retrieved height with its corresponding value in Table I, which means assuming those values as our ground truth.

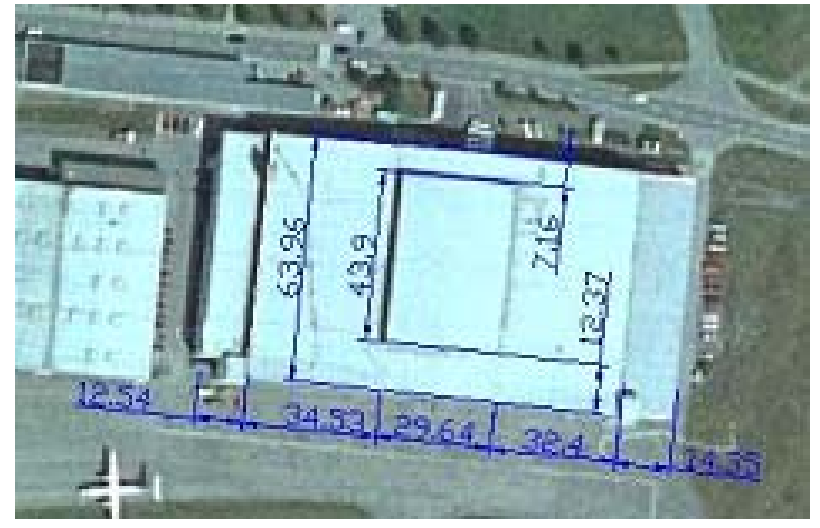

Figure 2. Enlarged view of the area in Fig.1 Lengths and widths of the building are in meters.

TABLE I. BUILDING HEIGHTS

\begin{tabular}{|l|l|r|}
\hline \multirow{2}{*}{ Long side } & Central part & $14.12 \mathrm{~m}$ \\
\cline { 2 - 3 } & Upper part & $3.25 \mathrm{~m}$ \\
\hline \multirow{3}{*}{ Short side } & Lower part & $8.00 \mathrm{~m}$ \\
\cline { 2 - 3 } & Central part & $5.00 \mathrm{~m}$ \\
\cline { 2 - 3 } & Upper part & $3.25 \mathrm{~m}$ \\
\cline { 2 - 3 } & & \\
\hline
\end{tabular}

As anticipated, information on materials has been also collected visiting the site and the relevant dielectric constants have been calculated, for the particular frequency of the SAR image, according to some empirical models adopted in literature [5].

\section{FEATURE EXTRACTION FROM REAL SAR IMAGES}

We can now move to inspect the SAR image of the scene in Fig.1. It is shown in Fig. 3. Directions of ground range $y$ and azimuth $x$ are also indicated. This type of image really represents a prototype of the products that will be available with the launch of new sensors. In fact, it is a high resolution SAR image $(1.99 \mathrm{~m}$ in range $x 1.80 \mathrm{~m}$ in azimuth) acquired on $20 / 04 / 2004$ by an airborne SAR sensor during a flight campaign in the area of Oberpfaffenhofen.

It has been obtained with the radar working in X-band $(9.6 \mathrm{GHz})$, a look angle of $55^{\circ}$ and using vertical polarization for both transmitting and receiving modes.

Actually, the original SAR image is a 16-bit image which means 65536 grey levels but showing the image in its original format is not useful as the most of contributions are in the low scale of the relative histogram. So, the 16-bit image would appear completely dark.

In order to see something in the image, we processed it with a byte scale operation in which we chose to represent the first 2000 grey levels of the original image with 256 levels. The image in Fig. 3 is the result of this elaboration.

Obviously, this has been done only to visualize the image because for the height retrieval, which is based on radiometric parameters, we exploited all the radiometric resolution working on the original image.

Now, applying considerations in [4] to each main part of the building satisfying the geometry of a parallelepiped, we should expect two double reflections lines from the long side and three from the short one. Instead, looking inside Fig.3, we find more lines than expected. This has been verified also by analyzing differently byte scaled versions of the SAR image.

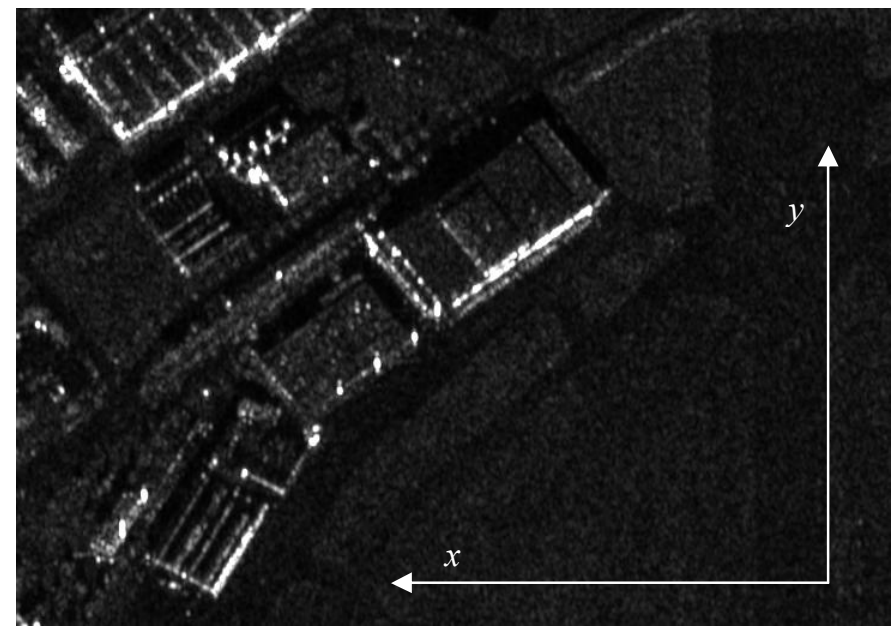

Figure 3. Byte-scaled SAR image relevant to the scene in Fig.1. (Courtesy of DLR) 
A possible explanation could be the following: if we consider, for example, the long side of the building, see Fig.4, the main façade is divided in two parts, the lower indented with respect to the upper, and this particular geometry could be responsible of the appearance of two close double reflection lines on the SAR image, each one corresponding to a portion of the total height. But this hypothesis can be simply shown to be false by measuring the distance between the lines and comparing it with the value expected by geometric considerations: this one is too small to be appreciated by the image resolution while, in Fig.3, the lines are clearly separable.

In this paper we will not discuss the interesting nature of these contributions, at the moment under study, being not our aim. But we took them into account in a first analysis in which we wanted to discriminate the lines due to double reflections from the others.

We did that, again, by means of geometrical considerations. In fact, every double reflection contribution can be seen as coming from the base of the wall forming the dihedral with the ground. Knowing the relative positions of all dihedrals in the building in Fig.2, also the double reflections lines can be localized without any doubt.

After having localized the right lines on the byte-scaled SAR image we moved to the extraction of the corresponding grey values in the original 16-bit image. For each line, a mean grey value has been computed considering the brightest pixels and their closest neighbours. This cutting operation has been carried out manually with the support of IDLiTools, in particular for the short side of the building to which we applied the extraction procedure.

We measured the brightness values $\mathrm{I}_{\mathrm{L}}=1452.75$, $\mathrm{I}_{\mathrm{C}}=1116.06$ and $\mathrm{I}_{\mathrm{U}}=865.00$ for the lower, the central and the upper part of this side, respectively. The values $\mathrm{I}_{\mathrm{C}}$ and $\mathrm{I}_{\mathrm{U}}$ have been employed, together with the information on the height of the central and upper part of the short side in Table 1, to calculate the calibration constants. For this operation, see (1), also information collected in situ as explained in the previous section has been considered together with the radar parameters and the wall orientation $\varphi=55^{\circ}$ extracted by the SAR image. In this way the element of the scattering matrix $S_{V V}$ has been computed and we found $\left|S_{V V}\right|=0.495509$.

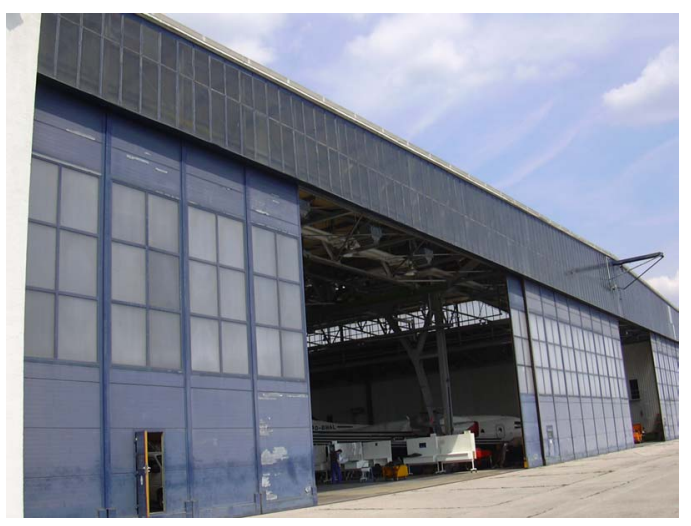

Figure 4. Long side of the building in Fig.2
Once that all parameters in (1) were known, we moved to the extraction of the height of the lower part of the short side starting from the knowledge of $\mathrm{I}_{\mathrm{L}}$. With the explained procedure, that part of the wall has been evaluated to be $7.93 \mathrm{~m}$ high, with a difference of only $7 \mathrm{~cm}$ with respect to the value of $8 \mathrm{~m}$ extracted by the optical image. The height retrieved is completely independent from the image resolution.

Some comments are in order. The obtained result is certainly very good but this is only a first experiment and the procedure needs to be tested on many other buildings to be assessed. Its application at the heights of three different parts of the same building allowed the authors to go around problems concerning the imperfect knowledge of some parameters as described in [3]. For example, the same wall orientation $\varphi$ has been measured and assumed for all three dihedrals and this leads to disregard possible errors coming from a not well described dependence between the radar cross section and the wall orientation. Application to buildings with different orientations is at the moment under study.

The main problem faced by the authors remains the need of a high a-priori knowledge of the scene. But the sensitiveness analysis led in [3] has been employed to partly overcome this matter.

\section{CONCLUSIONS}

The deterministic approach for feature extraction has been here applied to a real high resolution SAR image. For the first time in literature, a building height has been retrieved from radiometric parameters measurable on the SAR image with good results. High a-priori knowledge of the scene remains a necessary requirement. The procedure needs to be applied to many other examples to be further assessed.

\section{ACKNOWLEDGMENT}

The authors thank Ms. Karin Hedman, M.Sc., for her precious support during the visit of the site.

\section{REFERENCES}

[1] A.Roth, R.Werninghaus, "Status of the TerraSAR-X Mission", Proceedings of the International Geoscience and Remote Sensing Symposium, Denver (Colorado, USA), pp.1918-1920, 2006.

[2] G.Franceschetti, R.Guida, A.Iodice, D.Riccio, G.Ruello, "Deterministic Extraction of Building Parameters from High Resolution SAR Images", Proceedings of the $3^{\text {rd }}$ RSS/ISPRS joint Symposium on Remote Sensing and Data Fusion over Urban Areas, Tempe (Arizona, USA), 2005.

[3] G.Franceschetti, R.Guida, A.Iodice, D.Riccio, G.Ruello, "Accuracy of Building Height Estimation from SAR images", Proceedings of the International Geoscience and Remote Sensing Symposium, Denver (Colorado, USA), pp.3647-3650, 2006.

[4] G.Franceschetti, A.Iodice, D.Riccio, "A Canonical Problem in Electromagnetic Backscattering from Buildings", IEEE Transactions on Geoscience and Remote Sensing, vol.40, no.8, pp.1787-1801, 2002.

[5] E.J.Jaselskis, J.Grigas, A.Brilingas, "Dielectric Properties of Asphalt Pavement", Journal of Materials in Civil Engineering, vol.15, issue 5, pp.427-434, 2003 